\title{
Editorial
}

\section{Role of Loop Electrosurgical Excision Procedure (LEEP) in the Management of Cervical Intraepithelial Neoplasia (CIN)}

Cervical intraepithelial neoplasia (CIN) is the preinvasive lesion of invasive cervical cancer. The natural history of development of CIN and its transformation to invasive cancer has been extensively studied and well understood. The appropriate management of women with $\mathrm{CIN}$ is a critical component and the basis of cervical cancer prevention program.

$\mathrm{CIN}$ is a relatively common problem, especially in women of reproductive age. Laboratory surveys from the mid 1990s from the college of American pathologists suggest that more than 1 million women are diagnosed each year with low grade cervical intraepithelial lesion or CIN I and that approximately 500,000 are diagnosed with high-grade cervical intraepithelial lesion or CIN II, CIN III in USA ${ }^{1}$. According to the annual report of the Department of Pathology of Bangabandhu Sheikh Mujib Medical University, total 680 CIN were diagnosed in 2012. Among them CIN I was $75.6 \%$, CIN II was $20.7 \%$ and CIN III was $3.7 \%^{2}$.

$\mathrm{CIN}$ is most commonly detected in women in their 20s; the peak incidence of carcinoma in situ cervix is in women aged 25-35, while the incidence of cervical cancer arises after the age of $40^{3}$.

CIN is a histological diagnosis done by histopathological examination of a punch biopsy specimen obtained from aceto white area during colposcopic examination. Alternatively multiple punch biopsy taken from VILLI positive area can also diagnose various degrees of $\mathrm{CIN}$.

The treatment for CIN (Cervical intraepithelial neoplasia) depends on whether it is grade 1,2 or 3 . Various grades of CIN (CIN I, II and III) are graded according to the involvement of varying degrees of thickness of stratified squamous epithelium of ectocervix.

Women are separated into 3 groups on the basis of the biopsy report. a) Low grade squamous CIN (CIN I)

b) High grade squamous CIN (CIN II and CIN III).

c) Cervical glandular intraepithelial neoplasia (CGIN) including adenocarcinoma in situ (AIS).

Often cells showing CIN I return back to normal without any treatment at all. The aim of treatment for CIN II and III is to remove the abnormal area or destroy the abnormal cells while causing as little damage as possible to surrounding healthy tissues. There is no accepted nonsurgical therapy for $\mathrm{CIN}^{4}$.

The treatment modalities for preinvasive cervical disease or CIN are (A) Ablative procedures which include cryotherapy and $\mathrm{CO}_{2}$ laser vapourization (B) Excisional procedures, including LEEP, cold knife conization, $\mathrm{CO}_{2}$ laser excision and hysterectomy. A Cochrane Database of Systematic Review (2003) examined surgical treatment modalities for cervical intraepithelial neoplasia ${ }^{5}$. The evidence from 28 randomized controlled trials suggested that there is no overwhelmingly superior technique for eradicating CIN. Cryotherapy is an effective treatment for CIN I or LSIL but not for CIN II, III or HSIL ${ }^{5}$. Another author of India believes that ablation is not suitable for treating high grade squamous or glandular cervical intraepithelial lesion, as the depth of destruction would not be adequate 6 .

A Group of authors of a handbook for Gynecologic oncology in USA suggest that randomized controlled clinical trials comparing cryotherapy laser ablation and LEEP for treating biopsy confirmed CIN have reported no significant differences in either complication rates or success rates. Before treating any grade of $\mathrm{CIN}$ using an ablating modality such as cryothrapy, it is important to perform an endocervical sampling (ECC) in order to assure that an unsuspected lesion is not present in the endocervical canal ${ }^{7}$. On the other hand, such type of ECC is not required while treating CIN by excision method like LEEP. LEEP is the preferred method for treatment of CIN II and III. It is the most common method for removing abnormal cells from 
cervix. It takes 5 to 10 minutes, done under local anesthesia as an outpatient procedure.

Cortier originally developed an electrosurgical method for management of CIN using $5 \mathrm{~mm}$ rectangular, thin wire loops to sample and treat the cervix by removing the epithelium and underlying stroma in multiple $5 \mathrm{~mm}$ strips $^{8}$. The process was time consuming and thermal injury at the edge of the strips frequently compromised the specimen.

Prendiville et al 1990 introduced larger loop electrodes, 1 to $2 \mathrm{~cm}$ in width and $0.7-1.5 \mathrm{~cm}$ in depth, for excision of the entire transformation zone, usually in a single pass ${ }^{9,10}$. The combination of very thin wire loops and modern electrosurgical generators capable of delivering high powers (35 to $55 \mathrm{w}$ ) has allowed electrosurgical cutting with little associated thermal injury ${ }^{11}$.

Complications are minimal, comparing favorably with those associated with $\mathrm{CO}_{2}$ laser procedures ${ }^{5}$. Postoperative bleeding occurs in $2 \%$ to $5 \%$ of patients. Postoperative infection rate is $5 \%$ to $10 \%$. Clinically significant cervical stenosis and cervical incompetence are rare complications, but the patient must be made aware of the possibility of such adverse reproductive sequelae. Cure rates are comparable with those achieved $\mathrm{CO}_{2}$ laser procedures and with "cold-knife" conization, often in excess of $95 \%{ }^{5}$.

Electrosurgical loop excision offers several advantage over $\mathrm{CO}_{2}$ laser ablation. The procedure is quicker and easier. However, ease of use carries an attendant risk of overuse. Patient acceptance is improved and intraoperative pain is decreased. The submission of the entire specimen for histologic study increases the probability that unsuspected cancer will be detected and not ablated. In many large studies of LEEP, the unsuspected invasive cancer and highgrade glandular disease rate has been as high as $1 \%$ to $2 \%$ (12-15).

The role of LEEP in the management of CIN can be summarized as follows -

1. There is a definite role of LEEP in biopsy confirmed CIN II and CIN III where a diagnostic and therapeutic excision procedure is recommended for all women?

2. In case of unsatisfactory colposcopic examination report but the woman is symptomatic LEEP procedure is recommended.
3. In patient with recurrent CIN ( CIN I, CIN II or CIN III), it is preferred that an excisional treatment modality be used.

4. When pathologic discrepancy arises between high-grade cytology on papanicolaou (Pap) smear and low-grade histology on cervical biopsy, LEEP is one of the management alternative. In nonadolescent patients, a LEEP procedure is a reasonable management option for discrepancy between pap's and colposcopically directed biopsy ${ }^{16}$.

5. Any glandular lesion diagnosed by cytology is also an indication for LEEP or cold knife cone biopsy.

6. It should be noted that the risk of having an undetected CIN II, CIN III or adenocarcinoma in situ lesion is expected to be greater in women with CIN I preceded by a HSIL or atypical glandular cells of undetermined significance (AGUS) cytology result than for women with CIN I preceded by an ASCUS or LSIL cytology result. CIN II and CIN III is identified in 84-97\% of women with HSIL cytology evaluated using a loop electrosurgical excision procedure. Therefore, in the 2006 guidelines of American society for colposcopy and cervical pathology, separate recommendations are made for women with $\mathrm{CIN}$ I preceded by an HSIL or AGUS cytology result.

The advantages of LEEP over destructive methods of treatment have been detractive by several authors-

1. It allows for histologic audit of the colposcopic diagnosis.

2. It allows histopathology examination to rule out micro invasion.

3. It allows excision of the dysplastic lesion and the transformation zone, which may be confirmed histologically.

4. It may be performed at the first (assessment) colposcopic examination.

5. It may be adapted to treat all cases of CIN, irrespective of the size and site of the transformation zone.

6. It is an easily learned technique.

7. It uses inexpensive, readily available equipment and has low operating costs. 
8. It is usually an office or outpatient procedure performed using local anesthesia. The last five of these eight advantages also potential disadvantages of the technique that may combine to increase the morbidity of the procedure.

Another potential advantage of LEEP is the ability to "see-and-treat" at one visit. However, histologic study of loop-excised specimens removed at "see-and-treat" approach revealed no disease in $5 \%$ to $40 \%$ of specimen, particularly in young women referred with minor cytologic abnormalities ${ }^{17}$. Economic burden, overtreatment and complication of "see-and-treat" is also a concern. So, it is not applicable for our women.

\section{Prof. Sabera Khatun}

Professor of Gynae Oncology

Department of Obs \& Gynae

Bangabanghu Sheikh Mujib Medical University

Chairman, Oncology Task Force, OGSB.

\section{References:}

1. Davey DD, Neal MH, Wilbur DC, Colgan TJ,Styer PE, Mody DR. Bethesda 2001 implementation and reporting rates: 2003 practices of participants in the College of American Pathologists Interlaboratory Comparison Program in Cervicovaginal Cytology. Arch Pathol Lab Med 2004; 128:1224-9.

2. Annual report,Department of Pathology, Bangabandhu Sheikh Mujib Medical University 2012.

3. Christine H. Holschneider, MD., 2003. Premalignant \& Malignant Disorders of the uterine cervix. In: Alan H. DeCherney, MD , Lauren Nathan, MD. Editors. Current Obstetric \& Gynaecologic Diagnosis \& Treatment. $9^{\text {th }}$ edition. The McGraw-Hill Companies, PP.894.

4. Bell MC, Alvarez RD. Chemoprevention and vaccines: a review of the nonsurgical options for the treatment of cervical dysplasia. Int J Gynecol Cancer 2005; 15:4-12.

5. Martin-Hirsch PL, Paraskevaidis E, Kitchener H. the Cochrane Database of Systematic Reviews. Surgery for cervical intraepithelial neoplasia. The Cocherane Library 2003;3: 1:1-40.

6. B Shakuntola Baliga., 2008. Step by Step Colposcopy Cryosurgery and LEEP $.1^{\text {st }}$ edition. Jaypee Brothers Medical Publishers, New Dilhi, pp. 132.
7. Douglas A. Levine, JenniferDe Los Santos, Gini Fleming, Richard R. Baraket, Maurie Markman, Marcus E. Randall, 2010. Handbook for Principles and Practice of Gynaecologic Oncology.Lippincott Willims \& Wilkins, PP.69, 70.

8. Cartier R. The role of colposcopy in the diagnosis and treatment of dysplasia and intraepithelial carcinomas of the uterine cervix. Bull Cancer 1979; 66:447-454.

9. Prendiville W, Cullimore J. Excision of the transformation zone using the low voltage diathermy (LVD) loop: a superior method of treatment. Colpose Gynecol Laser surg 1987; 122S:1-15.

10. Prendiville W, Cullimore J, Norman S. Large loop excision of the transformation zone (LLETZ): a new method of management for women with cervical intraepithelial neoplasia. BJOG 1989; 96:1054-4060.

11. Jonathan S. Berek, Neville F. Hacker, 2010.Gynecologic Oncology $5^{\text {th }}$ edition. Lippincott Willims \& Wilkins, PP. 309.

12. Phipps JH, Gunasekara PC, Lewis BY. Occult cervical carcinoma reveled by large loop diathermy. Lancet 1989;453-454.

13. Chapptte OA Bryne DL, Raju KS, Nayagam M, Kenny A. Histological differences between colposcopic-directed biopsy and loop excision of the transformation zone (LLETZ): a cause for concern. Gynecol Oncol $1991 ; 43: 46-50$.

14. Murdoch JB, Grimshaw RN, Morgan PR, Monaghan JM. The impact of loop diathermy on management of early invasive cervical cancer. Int J Gynecol Cancer 1992; 2:129-133.

15. Burger MPM, Holema $\mathrm{H}$. The reliability of the histologic diagnosis in colposcopically directed biopsies: a plea for LLETZ. Int J Gynecol Cancer 1993; 385-390.

16. Sue L. Moreni, Caroline M. Mitchell, Rochelle L. Garcia and Linda O. Eckert, Obstetrics and Gynecology International Volume 2010 (2010), Article ID 743097, 6 pages doi:10.1155/2010/ 743097.

17. Howells REJ, O'Mahony F, Tucker H, Millinship $\mathrm{J}$, Jones PW, Redman CWE. How can the incidence of negative specimen resulting from large loop excision of the cervical transformation zone (LLETZ) be reduced ? An analysis of negative LLETZ specimens and development of a predictive model. BJOG 2000;107;1075-1082. 